\title{
THE EFFECT OF INTERNAL ENVIRONMENT OF ANIMALS ON PROPERTIES OF PROBIOTIC BACTERIA
}

\section{O. Aheyev}

From the very beginning of microbiological science an idea of the depth of microorganism interaction with its microflora was limited for a long time to the confidence that normocenosis representatives in all their diversity live exclusively on the surfaces of a macroorganism, mainly on the epithelium of the gastrointestinal tract (GIT). The presence of any microorganisms in blood, and even more so - in the tissues of parenchymal organs was a priori interpreted solely as the sign of progressive pathologic state and (or) as a result of a substantial weakening of organism protective capacity [1].

Only in the late twentieth century, mostly in foreign scientific literature, there appear more experimental data that contradicted with the traditional idea of the mechanisms of probiotics action on animals and humans. The authors from different countries noted instances of probiotic preparations efficacy that were are not subject to explanation in terms of conventional vision - not only to treat infections of the digestive tract at the intestine colonization, but also in the treatment of pathological processes located outside the GIT, namely: external cover injuries, postoperative suppurations, pyelonephritis, respiratory diseases, etc. [2; 3].

The discovered phenomenon of bacterial penetration into blood and parenchymal organs through the uninjured wall of the gastrointestinal tract was called "bacterial translocation" and was first described by American researcher R D. Berg in 1985 [4].

At the beginning of the second millennium, V. I. Nikitenko et al. [5], with the help of two strains of hay bacillus radiolabeled with $\mathrm{H}^{3}$-leucine, proved the possibility of probiotic bacteria translocation from the gastrointestinal tract into blood and lymph flow. According to V. I. Nikitenko [6] V. V. Smirnov et al. [7], V. A. Kopylov, V. V. Zakharov [8], L. de Souza et al. [9] the translocation may be a natural defense mechanism conditional to microorganism functions and not bacteria properties. Thus, there is evidence, that probiotic strains bacterial translocation and short-term asymptomatic bacteraemia, caused by it, can be one of important factors for activation and maintenance of nonspecific resistance of microorganism itself on a sufficient level.

Today the development of new approaches to the strategy and tactics of creation and use of biological preparations of bacterial origin, including probiotics, is urgent. And due to the comprehensive research of mutual influence of macroorganism and probiotic bacteria it becomes possible to create probiotic veterinary and medical preparations intended for the prevention and treatment of not only digestive system disorders, but many diseases of farm animals and humans, localized outside gastrointestinal tract [10-12].

Materials and methods. Probiotic strains of Bacillus subtilis 44-p (the basis of BPS-44 preparation), B. subtilis B3, Lactobacillus plantarum L5 (part of BPS-L preparation), L. plantarum Lc-18 (perspective) were used in the study. Pathogenic test cultures Staphylococcus aureus 23, Escherichia coli 14/1, Salmonella typhimurium 12, Shigella sonnei 22 from the collection of the Probiotics Laboratory of the Institute of Agricultural Microbiology and Agroindustrial Manufacture of NAAS were used to determine the antagonistic activity.

The cultivation probiotic strains of aerobic bacilli and pathogenic test-cultures (for the determination of antagonistic activity) was performed on standard nutrient media meat-peptone broth (MPB) and meat-peptone agar (MPA); lactic acid bacteria - on De Man medium (MRS) [13]. Daily culture was used for studies of bacterial translocation.

The investigation of the influence of translocation process on culturalmorphological and physiological and biochemical properties of bacteria-probiotics was conducted on laboratory rabbits. Groups of animals were formed for that - one control and four experimental (by the number of investigated strains), 5 animals in each group. 
Before the experiment, blood from all animals was taken and bacteriologically examined for the presence of aerobic bacilli and lactic acid bacteria in it.

The investigated strains of bacteria were administered to animals per os, once, with water at the dose of $3 \times 10^{8} \mathrm{CFU}$, drinking water was administered to control animals.

The separation of bacteria from the internal environment of experimental animals was conducted in the moments of their greatest accumulation in blood that had been found during previous studies. For aerobic bacilli that period is $5-10 \mathrm{~min}$. from the moment of their oral administration; for lactic acid bacteria - 12-24 hours. Pure cultures were isolated by limiting dilution using appropriate culture media, inoculation was performed from the dilutions of $10^{\circ}$ (native material) to $10^{-8}$ (to obtain isolated colonies).

Housing, feeding, care and all manipulations with laboratory animals were carried out under the European Convention "For the Protection of Vertebrate Animals used for Experimental and other Scientific Purposes" (Strasbourg, 1986) [19] and "General Ethical Principles of Experiments on Animals" approved at the first National Congress on Bioethics (Kyiv, 2001) [20].
Experiments were conducted in compliance with the principles of humanity set out in the European Community Directive [21].

Cultural-morphological (morphology of cells and colonies, peculiarities of the growth on nutrient media) and physiological and biochemical (exoenzymes activity, the ability to ferment various sources of Carbon, the amount of formed acid, antagonistic activity) properties were studied in the initial probiotic strains and cultures isolated from blood and organs of experimental animals [14-18].

Mathematical processing of all obtained results that are quantified, were done by the methods of variation statistics using Student t-test and correlation coefficients [22].

So, the translocation process of researched probiotic lactic acid bacteria into the internal environment of warm-blooded animals does not cause such changes in cultural-morphological characteristics, physiological and biochemical properties of bacteria that could be detected with the help of used methods; it can be explained by greater stability of their phenotype determined during phylogenesis, and higher heat resistance. 